\title{
A complementary metal-oxide-semiconductor compatible monocantilever 12-point probe for conductivity measurements on the nanoscale
}

Gammelgaard, Lauge; Bøggild, Peter; Wells, J.W.; Handrup, K.; Hofmann, Ph.; Balslev, M.B.; Hansen, J.E.; Petersen, P.R.E

Published in:

Applied Physics Letters

Link to article, DOI:

$10.1063 / 1.2888746$

Publication date:

2008

Document Version

Publisher's PDF, also known as Version of record

Link back to DTU Orbit

Citation (APA):

Gammelgaard, L., Bøggild, P., Wells, J. W., Handrup, K., Hofmann, P., Balslev, M. B., Hansen, J. E., \& Petersen, P. R. E. (2008). A complementary metal-oxide-semiconductor compatible monocantilever 12-point probe for conductivity measurements on the nanoscale. Applied Physics Letters, 93(9), 093104. https://doi.org/10.1063/1.2888746

\section{General rights}

Copyright and moral rights for the publications made accessible in the public portal are retained by the authors and/or other copyright owners and it is a condition of accessing publications that users recognise and abide by the legal requirements associated with these rights.

- Users may download and print one copy of any publication from the public portal for the purpose of private study or research.

- You may not further distribute the material or use it for any profit-making activity or commercial gain

- You may freely distribute the URL identifying the publication in the public portal 


\title{
A complementary metal-oxide-semiconductor compatible monocantilever 12-point probe for conductivity measurements on the nanoscale
}

\author{
L. Gammelgaard, ${ }^{1, a)}$ P. Bøggild, ${ }^{1}$ J. W. Wells, ${ }^{2}$ K. Handrup, ${ }^{2}$ Ph. Hofmann, ${ }^{2}$ M. B. Balslev, ${ }^{3}$ \\ J. E. Hansen, ${ }^{3}$ and P. R. E. Petersen ${ }^{3}$ \\ ${ }^{1}$ DTU Nanotech-Department of Micro- and Nanotechnology, Technical University of Denmark, Denmark \\ ${ }^{2}$ Institute for Storage Ring Facilities and Interdisciplinary Nanoscience Center (iNANO), \\ University of Aarhus, Denmark \\ ${ }^{3}$ Capres A/S, 2800 Kgs Lyngby, Denmark
}

(Received 11 July 2007; accepted 6 February 2008; published online 3 September 2008)

\begin{abstract}
We present a complementary metal-oxide-semiconductor compatible, nanoscale 12-point-probe based on TiW electrodes placed on a $\mathrm{SiO}_{2}$ monocantilever. Probes are mass fabricated on $\mathrm{Si}$ wafers by a combination of electron beam and UV lithography, realizing TiW electrode tips with a width down to $250 \mathrm{~nm}$ and a probe pitch of $500 \mathrm{~nm}$. In-air four-point measurements have been performed on indium tin oxide, ruthenium, and titanium-tungsten, showing good agreement with values obtained by other four-point probes. In-vacuum four-point resistance measurements have been performed on clean $\mathrm{Bi}(111)$ using different probe spacings. The results show the expected behavior for bulk $\mathrm{Bi}$, indicating that the contribution of electronic surface states to the transport properties is very small. () 2008 American Institute of Physics. [DOI: 10.1063/1.2888746]
\end{abstract}

The linear four-point probe is a standard instrument for measuring the resistivity of semiconductors and other materials, both for the bulk and for thin films (for a sketch see Fig. 1). It offers an alternative to the well-known van der Pauw geometry ${ }^{1}$ to achieve a flexible, local, and accurate measurement. Four-point probes have been scaled down to sizes of a few micrometers ${ }^{2}$ and applied for investigations on a wide range of materials. ${ }^{3-5}$

The standard microscopic four-point probe, which consists of four individual Au-coated $\mathrm{SiO}_{2}$ cantilevers, cannot meet the recent demands of the semiconductor industry for small pitched complementary metal-oxide-semiconductor (CMOS) compatible probes. In order to meet the criteria of CMOS compatibility, the probes cannot contain metals or silicides with high mobility, since such materials could greatly affect the properties of the investigated samples. In this paper, we present an approach to the fabrication of fourpoint probes which fulfills this requirement. Moreover, this approach pushes the miniaturization to the nanometer scale; another important feature for many current and future applications, including measurements on magnetic tunnel junction stacks to determine the magnetoresistance and resistance area product and, of course, any measurement on a true nanostructure. ${ }^{6,7}$

A four-point probe can be used for a resistance measurement by passing a current through two of the four tips, usually the two outermost, and measuring the voltage drop across the two other tips. If the voltage measurement is performed without draining any current, the sample's resistance can be measured completely independent of the contact resistance or even of the possible non-Ohmic nature of the contacts. In the case of a homogeneous, two-dimensional sheet of material, the four-point probe resistance $R$ is found to be

${ }^{\text {a)} E l e c t r o n i c ~ m a i l: ~ l a u g e g a m m e l g a a r d @ g m a i l . c o m . ~}$

$$
R=\frac{U}{I}=\frac{\ln 2}{\pi \sigma_{s}},
$$

where $U$ is the measured potential drop, $I$ the current, and $\sigma_{S}$ the sheet conductivity of the sample. ${ }^{8}$ Note that for the case of a two-dimensional sheet, the measured resistance is independent of the distances between the probe contacts, but this is not the case for measurements on semi-infinite bulk samples. Any thin film can be viewed as a two dimensional sheet of material if the thickness of the film is much smaller than that center to center distance of two neighboring tips of the four-point probe, i.e., the so-called pitch of the probe.

The smallest probe pitch which has been achieved with the currently most advanced construction principle of individual Au-covered $\mathrm{SiO}_{2}$ cantilevers is $1.1 \mu \mathrm{m}$, but it is hard to see how this design principle can be miniaturized even further. This is due to difficulties of controlling the necessary underetch of the cantilevers to prevent short circuits between the probe electrodes together with the increasing softness of the individual cantilevers that decrease the control of the probe pitch leading to inaccurate measurements. (Actually, this smallest pitch has not been realized on a four-point probe but as the smallest possible pitch on a probe with 12

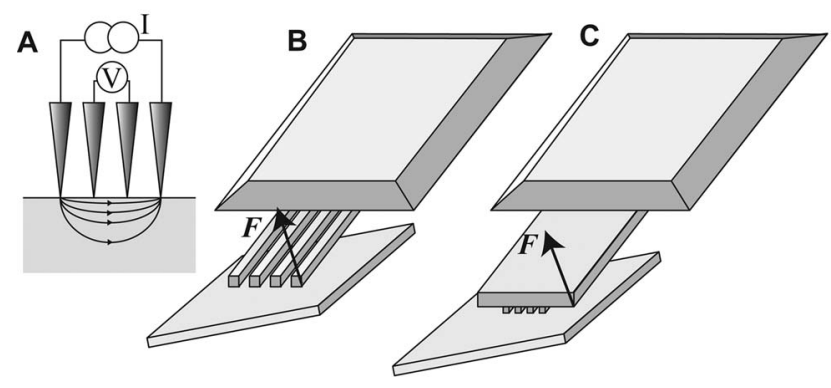

FIG. 1. (a) four-point measurement where a current is passed through the two outer probes and the voltage potential drop in the material is measured over the two inner electrodes. (b) Sketch of a four-point probe based on four individual cantilevers (commercially available from Capres A/S) in contact with a sample surface. The initial point of contact is shown as an arrow. (c) A monocantilever in contact with a sample surface. 
tips. Only four of these 12 tips are usually used at any time but the possibility to choose these four tips offers an effective way of changing the probe pitch.)

In addition to the pitch, other design parameters are important for the probes. The probes should be mechanically strong in order to cope with a slight misalignment. Ideally, the probe should be aligned parallel to the sample surface such that all tips make contact at the same time. In reality, a small misalignment is often present. This leads to a variation of contact pressure or can even cause the cantilevers to break. This problem can be alleviated by mounting all tips on one cantilever, ${ }^{9}$ an approach also used here (see Fig. 1).

A very precise control of the probe pitch is also necessary to avoid short circuiting of the individual tips or large uncertainties in pitch-size dependent measurements. ${ }^{10,11} \mathrm{Fi}-$ nally, and maybe most importantly, a suitable tip electrode material should be chosen. The ideal material should be conductive, CMOS-compatible, wear resistant, nonoxidizing, and compatible with the production of probes on Si wafers.

In this paper, we describe the fabrication and test of four- and 12-point probes with a pitch down to $500 \mathrm{~nm}$ and with electrodes made of TiW (TiW with 10/90 wt \%). The electrodes are placed on a microscale $\mathrm{SiO}_{2}$ support cantilever with a spring constant of around $0.5 \mathrm{~N} / \mathrm{m}$, making it comparable to contact mode atomic force microscope (AFM) cantilevers and conveniently allowing for self-aligning of the probe tips to the sample surface. The choice of TiW as electrode material is important. It does not create an insulating oxide, allowing good contact to the samples and it has a rather low resistivity of around $70 \mu \Omega \mathrm{cm}$ (depending on the deposition parameters). Also, it has a high wear resistance, improving probe lifetime, and it can be sputter-deposited stress-free. It has a high etch resistivity toward most standard etches used in clean room processing, and finally, it is CMOS compatible. A low surface roughness is also important to avoid large variations in the contact area of the electrodes. AFM measurements of TiW thin films have shown a surface roughness of 4-7 nm.

An outline of the fabrication process can be seen in Fig. 2. The fabrication of the four-point probes is based on a mix-and-match lithography strategy, where the electrode tips and overlap area are written with an e beam and the rest of the wires and contact pads are made by standard UVlithography. Initially, the electron beam lithography is made to ensure a completely flat, clean surface for spinning the e-beam resist, followed by lift-off of the deposited $50 \mathrm{~nm}$ thick TiW.

The wafers are then thoroughly cleaned in acetone, 7-up (sulfuric acid and ammoniumperoxodisulfate), and isopropanol and the UV lithography pattern is aligned to the TiW electrode tips, followed by sputtering of a $100 \mathrm{~nm}$ TiW layer. It is important to use a high ultrasonic power during lift-off to avoid leaving small bridges of TiW that can short circuit the probe wiring. Photoresist is then spun on the front side and the support cantilever is defined by careful alignment to the electron beam written TiW electrode tips with a standard $40 \%$ bHF etch. After an acceptable alignment has been achieved, the wafers are again cleaned in 7-up and rinsed in isopropanol. Next, the front and back side are encapsulated by a pinhole free, $220 \mathrm{~nm}$ low-stress plasma-enhanced chemical vapor deposition silicon nitride layer with a high $\mathrm{KOH}$ resistance developed for this purpose. The back side nitride coating is then patterned with UV lithography, and
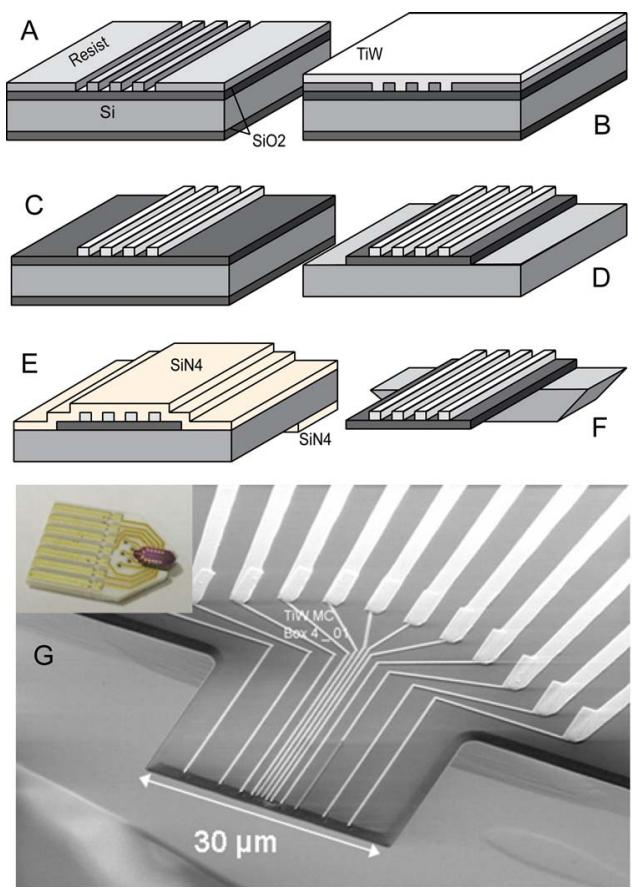

FIG. 2. (Color online) Outline of fabrication process. (a) A double polished silicon wafer with $1 \mu \mathrm{m} \mathrm{SiO}_{2}$ and patterned photoresist on top. (b) $\mathrm{TiW}$ is deposited. (c) Lift-off and lithography mix-and-match creates the electrode pattern. (d) The support cantilever is etched in bHF. (e) Encapsulation with $\mathrm{Si}_{3} \mathrm{~N}_{4}$ followed by opening of the backside with reactive ion etching. (f) The chip is defined with $\mathrm{KOH}$ and the support cantilever is released. (g) Scanning electron microscopy image of 12 -point probe with TiW electrodes placed on $\mathrm{SiO}_{2}$ cantilever and a probe pitch of $500 \mathrm{~mm}$ at the inner probes. They have an uneven spacing to increase the number of possible pitch configurations. Inset: The finished wire bonded 12-point probe mounted on a ceramic substrate.

opened by reactive ion etching. After $4 \mathrm{~h} 45$ min etching in $80{ }^{\circ} \mathrm{C} \mathrm{KOH}$, the chip body is defined with the cantilevers released.

After 10 min cleaning in $\mathrm{HCl}$, the silicon nitride is removed in a combination of reactive ion etching (selective toward TiW) and a 2 min etch in $160{ }^{\circ} \mathrm{C}_{3} \mathrm{PO}_{4}$. Finally, a shadow mask is used to deposit a $10 \mathrm{~nm} \mathrm{Ti}$ and $100 \mathrm{~nm} \mathrm{Al}$ layer on top of the bond pads to make wire bonding possible. The chips are then broken out of the support grid of the wafer, glued on a ceramic substrate, and wire bonded. An example of a fabricated 12-point probe after mounting on a ceramic substrate and wire bonding can be seen in Fig. 3 .

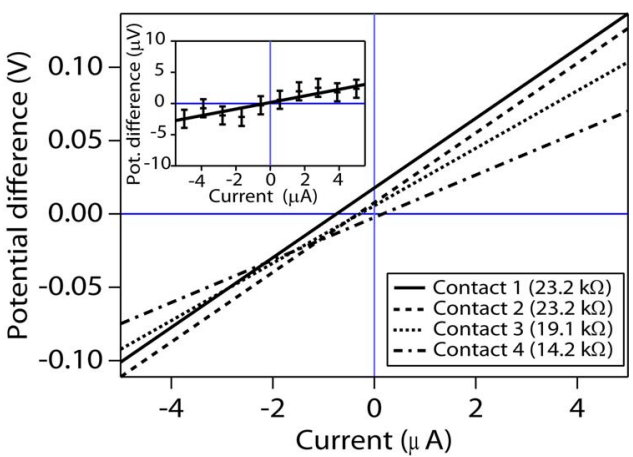

FIG. 3. (Color online) Four-point measurement performed on Bi(111) at room temperature. $I$ - $V$ curves of four different electrodes in contact with the Bi(111) surface demonstrating a good Ohmic contact. Inset shows $I-V$ fourpoint measurements made with T-shaped TiW probes with a pitch of 500 nm, see Fig. 1. 
TABLE I. Resistance of three thin films determined by the monocantilever TiW probes and by individual cantilever Au coated probes. All values in $\Omega$. The values are based on ten measurements.

\begin{tabular}{lccc}
\hline \hline & ITO & Ru & TiW \\
\hline TiW probes & $36.7 \pm 0.2$ & $1.7 \pm 0.1$ & $7.9 \pm 0.3$ \\
Au probes & $36.0 \pm 0.1$ & $1.6 \pm 0.1$ & $7.6 \pm 0.1$ \\
\hline \hline
\end{tabular}

The performance of these probes has been tested by resistance measurements on thin films. According to Eq. (1), using thin films has the advantage that for a given material, the resistance measured with such a probe should be equal to the resistance measured by a traditional probe with a larger pitch.

In-air measurements have been carried out with $750 \mathrm{~nm}$ pitch TiW probes on 100-nm-thick thin films of three different materials: indium tin oxide (ITO), ruthenium (Ru), and titanium-tugsten (TiW). All measurements were done using an alternating current. The measurements with the TiW probes were made at $11 \mathrm{~Hz}$ with a source current of $10 \mu \mathrm{A}$. A low frequency was chosen because of the rather high serial resistance of the long narrow TiW wires, which is on the order of 25-35 $\mathrm{k} \Omega$ depending on the specific probe configuration. All measurements have been carried out with an automated Capres four-point measurement setup, using automatic engages of the probe to the sample surface for multiple and fast measurements. The results are compared to data obtained using well-established Capres 12-point probes with 12 individual $\mathrm{SiO}_{2}$ cantilevers covered by a $100 \mathrm{~nm}$ electrode layer of $\mathrm{Ti}$ and $\mathrm{Au},{ }^{12}$ using the smallest possible probe pitch of $1.5 \mu \mathrm{m}$. This comparison is given in Table I, demonstrating a good agreement between the two different types of 12-point probes.

The TiW probe with a $500 \mathrm{~nm}$ pitch has also been used for measurements on the (111) surface of Bi single crystals. The surfaces of $\mathrm{Bi}$ (a semimetal) have recently attracted some attention because of their enhanced density of states at the Fermi level ${ }^{13}$ which makes it conceivable that the surface contribution to the conductance could be observed by nanoscale four-point probe measurements. Measurements in ultrahigh vacuum were performed using a system in Aarhus. ${ }^{3}$ The $\mathrm{Bi}(111)$ surface was prepared in situ by repeated cycles of $\mathrm{Ar}$ ion bombardment and annealing to $150^{\circ} \mathrm{C}$. The surface order was checked using low energy electron diffraction. The individual contact resistances were measured. The current source was connected between one probe and the substrate and was then linearly increased from -5 to $+5 \mu \mathrm{A}$ in $20 \mathrm{~s}$, while the voltage drop between the contact and the substrate was recorded (giving a so-called $I$ - $V$ curve). The current was then linearly decreased back to $-5 \mu \mathrm{A}$. This measuring cycle was repeated at least 100 times for each contact. By measuring using both a positive and negative current ramp and comparing the gradients, any charging effects would become apparent. When using such a slow ramp, no such effects were seen. The measurements were repeated using several similar probes and after several repeated surface preparations, and the measurements were found to be reproducible.

After ensuring that reliable contacts could be made, fourpoint measurements were also performed on the $\mathrm{Bi}(111)$ surface. The measurements were performed in a similar manner to the contact measurements and by a scheme according to that shown in Fig. 1(a), i.e., a standard four-point measurement with a pitch of $500 \mathrm{~nm}$.

The measured change in potential during the measurements was on the order of microvolts, thus a 1000 times voltage amplifier was required. The measurements suffered from significant electrical noise, thus the sampling was increased to 3000 samples per point. Once again, the entire measuring cycle was repeated many times.

Again, it is clear that the behavior is Ohmic. By considering many such data sets, the four-point resistance is found to be $0.39 \pm 0.07 \Omega$. The four-point resistance is related to the resistivity by $R_{4 \mathrm{pp}, \text { bulk }}=\rho /(2 \pi s)$. ${ }^{4}$ Using this relationship, our measured values corresponds to a resistivity of $120 \pm 20 \mu \Omega \mathrm{cm}$, which is consistent with the accepted bulk resistivity of $130 \mu \Omega \mathrm{cm} .{ }^{14}$ Thus, it can be inferred that the measurements are bulk sensitive-which, in turn, indicates that the surface resistivity must be greater than approximately $10 \Omega$ (otherwise the surface resistivity would dominate the measurement). This result does not contradict the notion of a strongly enhanced density of surface states at the Fermi level found by angle-resolved photoemission ${ }^{13}$ because the mobility of these surface states is not known. The mobility of the bulk carriers in $\mathrm{Bi}$, on the other hand, is very high; thus, it will be somewhat challenging to determine the surface conductivity.

In conclusion, we have developed a CMOS compatible nanoscale 12-point probe based on TiW electrodes on a single $\mathrm{SiO}_{2}$ support cantilever. Working probes have been fabricated with a probe pitch down to $500 \mathrm{~nm}$. The electron beam lithography, in principle, allows far smaller dimensions. How small the electrode pitch can be made will be investigated in the future. So far the yield of $250 \mathrm{~nm}$ pitch probes (having $125 \mathrm{~nm}$ wide electrodes) is not high enough to support real measurements. The $750 \mathrm{~nm}$ pitch probes have been used to measure the sheet resistance of ITO, Ru, and TiW at $11 \mathrm{~Hz}$, demonstrating stable measurements in good agreement with commercially available $\mathrm{Au}$ probes. The probes have also been used in ultrahigh vacuum, and the four-probe resistance of a $\mathrm{Bi}(111)$ sample was measured and found to be $0.39 \pm 0.07 \Omega$ for a $500 \mathrm{~nm}$ pitched probe. This is consistent with the accepted bulk resistivity, and thus, indicates that the contribution of electronic surface states to the conductance is small.

${ }^{1}$ L. J. Van der Pauw, Philips Tech. Rev. 20, 220 (1958).

${ }^{2}$ C. L. Petersen, T. M. Hansen, P. Bøggild, A. Boisen, O. Hansen, and F. Grey, Sens. Actuators, A 96, 53 (2002).

${ }^{3}$ J. W. Wells, J. F. Kallehauge, and Ph. Hofmann, J. Phys.: Condens. Matter 19, 176008 (2007).

${ }^{4}$ J. W. Wells, J. F. Kallehauge, T. M. Hansen, and Ph. Hofmann, Phys. Rev. Lett. 97, 206803 (2006).

${ }^{5}$ P. Bøggild, F. Grey, T. Hassenkam, D. R. Greve, and T. Bjørnholm, Adv. Mater. (Weinheim, Ger.) 12, 947 (2000).

${ }^{6}$ D. C. Worledge and P. L. Trouilloud, Appl. Phys. Lett. 84, 1695 (2004).

${ }^{7}$ S. Yoshimoto et al., Nano Lett. 7, 956 (2007).

${ }^{8}$ F. M. Smits, Bell Syst. Tech. J. 37, 711 (1958).

${ }^{9}$ D. Kjær, L. Gammelgaard, P. Bøggild, O. Hansen, P. R. E. Petersen, and J. E. Hansen, J. Micromech. Microeng. 17, 1910 (2007).

${ }^{10}$ R. Lin, P. Bøggild, and O. Hansen, J. Appl. Phys. 96, 2895 (2004).

${ }^{11}$ C. L. Petersen, D. Worledge, and P. R. E. Petersen, MRS Symposia Proceedings, edited by D. A. Bonnell, J. Piqueras, A. P. Shreve, and F. Zypma, No. 738 (Materials Research Society, Pittsburgh, 2003), pp. 157162.

${ }^{12}$ See: www.capres.com

${ }^{13} \mathrm{Ph}$. Hofmann, Prog. Surf. Sci. 81, 161 (2006)

${ }^{14}$ See: www.webelements.com. 